\title{
SARS-CoV-2 Detection with De Novo-Designed Synthetic Riboregulators
}

\author{
Illkay Çisil Köksaldı," Sıla Köse,, Recep Erdem Ahan," Nedim Hacıosmanoğlu,, Ebru Şahin Kehribar," \\ Murat Alp Güngen, Aliye Baştuğ, Bedia Dinç, Hürrem Bodur, Aykut Özkul, \\ and Urartu Özgür Şafak Şeker*
}

Cite This: Anal. Chem. 2021, 93, 9719-9727

Read Online

ACCESS | 네 Metrics \& More | 回 Article Recommendations | st Supporting Information

ABSTRACT: SARS-CoV-2 is a human pathogen and the main cause of COVID-19 disease, announced as a global pandemic by the World Health Organization. COVID-19 is characterized by severe conditions, and early diagnosis can make dramatic changes for both personal and public health. Low-cost, easy-to-use diagnostic capabilities can have a very critical role in controlling the transmission of the disease. Here, we are reporting a state-ofthe-art diagnostic tool developed with an in vitro synthetic biology approach by employing engineered de novo riboregulators. Our design coupled with a home-made point-of-care device can detect and report the presence of SARS-CoV-2-specific genes. The presence of SARS-CoV-2-related genes triggers the translation of sfGFP mRNAs, resulting in a green fluorescence output. The

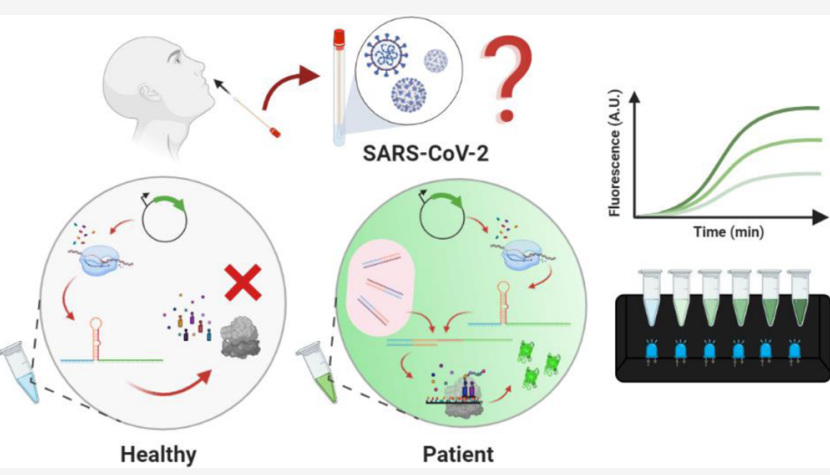
approach proposed here has the potential of being a game changer in SARS-CoV-2 diagnostics by providing an easy-to-run, low-cost diagnostic capability.

\section{INTRODUCTION}

Coronavirus disease 2019 (COVID-19) is a global pandemic caused by a novel coronavirus, severe acute respiratory syndrome coronavirus 2 (SARS-CoV-2), that infects angiotensin-converting enzyme 2 (ACE2) positive cells found in the human body. Several tissues and organ systems, including the upper respiratory tract, small intestine, liver, and nervous system, are susceptible to SARS-CoV-2 infection. ${ }^{1,2}$ As diverse clinical manifestations of COVID-19 are vaguely defined, the shared symptoms with other common infectious disease states such as the seasonal flu may cause misdiagnosis. ${ }^{3,4}$ Person-toperson transmission and, most importantly, the lack of rapid and adequate testing resulted in the loss of opportunity to prevent the spread of the virus in numerous countries at the beginning of the pandemic. Furthermore, the presence of asymptomatic and pre-symptomatic SARS-CoV-2-infected people, accounting for the silent transmission in superspreading events, aggravates the pandemic worldwide by posing a risk to elderly people and chronically ill patients. ${ }^{5}$

Test-track-isolate is a fundamental strategy to mitigate the risk of overwhelming the healthcare system until a viable therapeutic solution can be produced. ${ }^{6}$ Therefore, widespread testing for COVID-19 is vital to block the viral transmission chain. Molecular diagnosis of COVID-19 heavily relied on quantitative real-time polymerase chain reaction (qRT-PCR) that detects the presence of SARS-CoV-2 RNA in the nasopharynx, trachea, or bronchus. However, testing capacity failed to meet increasing demand due to the global shortage in qRT-PCR reagents (i.e., reaction mix, polymerase, reverse transcriptase, etc.). ${ }^{7,8}$ Additionally, qRT-PCR tests generally require a testing facility and trained staff. To compensate for the gap between test numbers and the pace of infection, many agencies declared an emergency use of in vitro diagnostic products for SARS-CoV-2. Consequently, numerous methods, excluding qRT-PCR, were developed for virus detection by targeting its proteins or nucleic acid components. ${ }^{9-12}$ Notably, CRISPR Dx tools, SHERLOCK(13) and DETECTR(14), were rapidly developed to sense SARS-CoV-2 presence with improvements in their sensing pipeline such as SHERLOCK with unextracted samples and amplification-free Cas13a sensing. Even though the limit of detection (LoD) of newly proposed tests is not as high as that of qRT-PCR, it is speculated that the point-of-care (PoC) device implementation will be useful to detect particularly people with high virus titer who are the main cause of super-spreading events. ${ }^{13}$

Received: February 26, 2021

Accepted: June 17, 2021

Published: June 30, 2021 
a) Toehold Mechanism

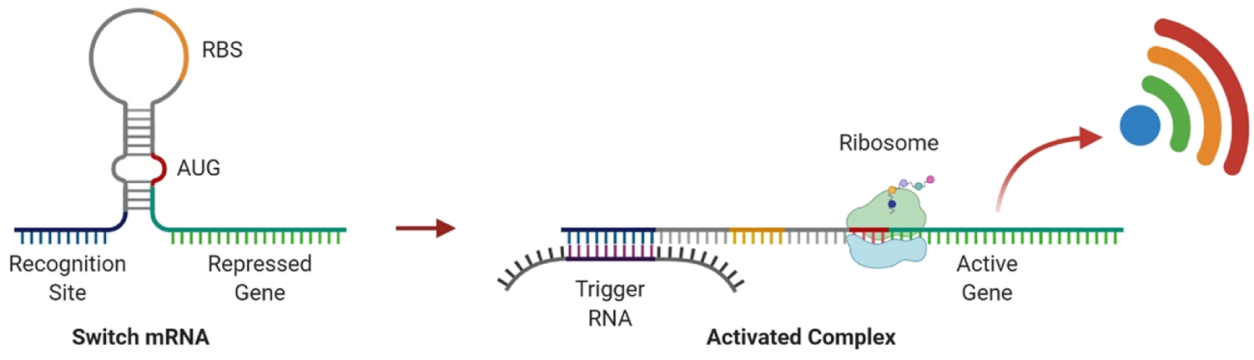

b) In Silico Sensor Design

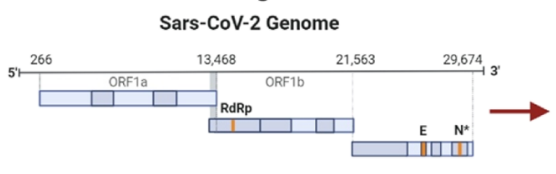

Candidate gene selection: compatible with toehold activation and NASBA amplification

c) In Vivo Sensor Validation

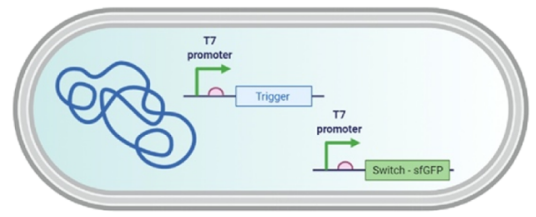

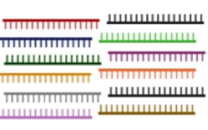

Generation of trigger candidates with NCBI: Primer BLAST

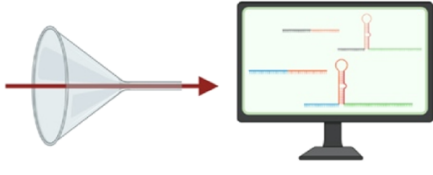

Elimination of unspesific and unfavorable candidates
Switch design with NUPACK

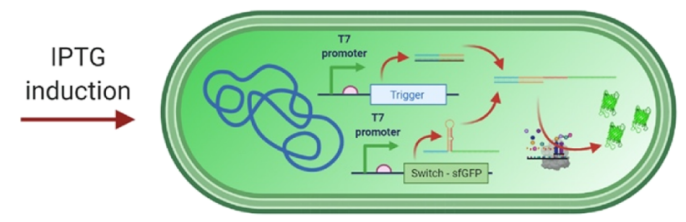

d) Cell-free System Optimization
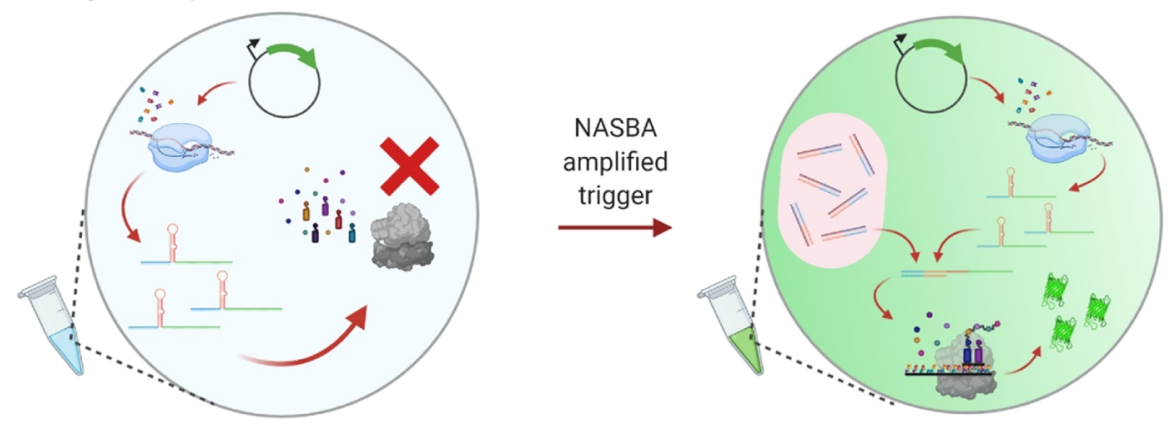

Figure 1. (a) Schematics of the operating principle of toehold biosensors. (b) In silico design of triggers and switches specific to the SARS-CoV-2 genome. Toehold triggers are generated using NCBI Primer BLAST. The trigger candidates that have non-optimal free energy predictions and candidates that are not compatible with species-specific amplification were eliminated. Eligible candidates were then used in the design of their respective toehold switches in silico using NUPACK software. (c) In vivo validation of designed toehold switches. Triggers were cloned downstream of the T7-LacO promoter. Switches were cloned downstream of the T7-LacO promoter and upstream of the sfGFP reporter gene. Both constructs were transformed into E. coli BL21 (DE3) cells. Cells that only have the switch plasmid and the cells that have both switch and trigger plasmids were induced with IPTG. (d) Optimization of NASBA and cell-free systems.

Synthetic biology broadens the scope of diagnostic tool alternatives, among which programmable riboregulator toehold switches are straightforward and inexpensive to develop. ${ }^{14}$ (16) The versatility of toehold switches enabled researchers to develop biosensors for pathogenic viruses such as Zika, ${ }^{15}$ Ebola, ${ }^{16}$ an antibiotic resistance gene, ${ }^{16}$ and microorganisms found in the human gut. ${ }^{17}$ Combined with the cell-free transcription/translation (TXTL) technology, toehold switches can be used to detect the presence of specific nucleic acid sequences in a PoC device in which the output signal can be a fluorescent protein or the colorimetric degradation product of an enzyme. ${ }^{15}$ Besides, the LoD of toehold-based sensors can be increased with a simple isothermal amplification reaction such as nucleic acid sequence-based amplification (NASBA) or recombinase polymerase amplification prior to the TXTL reaction. ${ }^{18}$ Owing to their fast prototyping capability and easy-to-use format, the utilization of the toehold switch sensor mechanism together with the TXTL reaction is an excellent candidate to develop an assay with. Such an assay can be implemented in a PoC device for surveillance of SARSCoV-2 in substantial portions of populations, which do not have access to a centralized facility to perform sophisticated diagnosis assays such as qRT-PCR or next-generation sequencing. Alongside mass testing, the need for such testing systems in less developed countries, where the necessary infrastructure and trained personnel are not always available, is paramount in preventing diseases from spreading. In order to help these countries, the World Health Organization (WHO) has developed the ASSURED (Affordable, Sensitive, Specific, User-friendly, Rapid and robust, Equipment-free, and Deliverable to end-users) criteria. ${ }^{19}$ Creating PoC devices that complies with these criteria increases the likelihood of better 
containing pandemics by using them in developing countries as well as by compensating for the growing need for testing in developed countries.

Here, we designed toehold switches to detect the presence of the SARS-CoV-2 virus. Specifically, regions in the SARSCoV-2 genome, ORF1ab, M, ORF678, and S coding sequences, those compatible with the NASBA reaction were selected as potential triggers. Switch sequences specific to their cognate triggers that control the expression of superfolder green fluorescent protein (sfGFP) were constructed and tested. Two out of four trigger sequences (the regions found in ORF $1 \mathrm{ab}$ and $\mathrm{S}$ proteins) were detected with designed switch sequences both in vivo on a genetic circuit and in vitro through TXTL reactions. Then, selected trigger/switch sensor couples are characterized for optimum conditions in NASBA and TXTL reactions to be used with real samples, as shown in Figure 1. Last of all, patient samples are tested with the designed system, and efficiency is compared with qRT-PCR analysis. In light of the results obtained, the promise of the toehold-based biosensors implemented in a PoC device to help monitor and control the transmission of the disease, especially in rural and less developed regions, has been demonstrated, thanks to the fact that it is cheap, accessible, and does not require expertise.

\section{MATERIALS AND METHODS}

In Silico Design. In order to develop an integrated workflow for designing NASBA primers and toehold switches together, a combination of web tools was used throughout the design procedure. For the generation of trigger sequence candidates with primer sites, Primer-BLAST was used (https://www.ncbi.nlm.nih.gov/tools/primer-blast/) by setting temperature parameters to $60{ }^{\circ} \mathrm{C}$ and PCR product size between 120 and $160 \mathrm{bp}$. Using the input gene sequences derived from the reference SARS-CoV-2 genome (GenBank: MN908947.3), we generated a set of trigger candidates with NASBA compatible primer sets for the whole SARS-CoV-2 reference genome. Next, we used a web-based analysis tool of nucleic acid package (NUPACK) software (http://www. nupack.org/) to investigate free energies of trigger candidates and applied a $-25 \mathrm{~kJ} / \mathrm{mol}$ constraint for selecting designs for the next step. Then, by using NCBI-BLAST web tool (https:// blast.ncbi.nlm.nih.gov/Blast.cgi), we aligned candidate sequences with six human coronaviruses (229E, NL63, OC43, HKU1, SARS, and MERS) to maximize specificity. Candidate triggers that passed energy and BLAST tests were transferred to the design phase by using the design tool of NUPACK software again to generate switch candidates. Ten candidate switches for each trigger candidate were designed. After completion of the designs, defects, similar sequences, and stop codon carrying constructs were eliminated by using a defect function threshold of $25 \%$. Overall, 503 trigger candidates were designed with Primer-BLAST, 123 candidates have passed the minimum energy and BLAST requirements, and five trigger candidates resulted in up to four different switch constructs. Toehold domains for each trigger targeting different regions in the SARS-CoV-2 genome contain $b^{*}, a^{*}$, conserved domain, a, and linker regions, as previously reported. ${ }^{18}$ The recognition domain consists of $b^{*}$ and $a^{*}$ regions; conserved domain; and a region creates the stem and loop domains. The lengths of domains are as follows: $b^{*}: 24 \mathrm{bp}, \mathrm{a}^{*}: 12 \mathrm{bp}$, conserved domain: 28 bp (containing 8 bp RBS and ATG sequences as well), a: $12 \mathrm{bp}$, and linker region: $21 \mathrm{bp}$. De novo-designed riboregulators specific to SARS-CoV2 are given in Table S1 (as the patent procedure is still in progress, exact sequences of these regions are not available. Once the procedure is finished, exact sequences of each trigger and switch sequences are going to be available).

Assembling Sensor Constructs. For the generation of switch and trigger plasmids, a pET22B backbone (high copy number, AmpR resistance) for triggers and a pZA backbone (mid copy number, $\mathrm{CmR}$ resistance) for switches were used as vectors. In our design, the pET22B backbone carries a T7 promoter and a T7 terminator, which can be induced by isopropyl- $\beta$-D-thiogalactoside (IPTG) addition. On the other side, pZA backbone carries a T7 promoter with sfGFP gene from the pJT119b plasmid ${ }^{20}$ (deposited to the Addgene by Jeffrey Tabor, Addgene \#50551) and a rrnB_T1 terminator from Registry of Standard Biological Parts at the downstream of sfGFP. Inserts selected during the in silico design phase were ordered from Genewiz Inc. with Gibson Assembly overlaps. Sequence verification of cloned constructs was performed with Sanger sequencing (Genewiz) after the cloning. DH5 $\alpha$ strain of Escherichia coli was used for all cloning stages.

Whole-Cell Sensor Test. As the first stage of characterizations, switch constructs with a matching trigger sequence were transformed into E. coli BL21(DE3) cells with chemical transformation. Meanwhile, standalone switch constructs were transformed as control groups. A single colony from each plate was selected and grown overnight in LB medium at $37^{\circ} \mathrm{C}$ in a $200 \mathrm{rpm}$ orbital shaker with appropriate antibiotics. Next, cells were diluted in fresh LB medium with antibiotics and induced with $1 \mathrm{mM}$ IPTG at an $\mathrm{OD}_{600}$ value between 0.2 and 0.3 . Total fluorescence emission of the trigger plasmid carrying cells and control groups was measured using a M5 SpectraMax (Molecular Devices) at 2, 4, 8, and $16 \mathrm{~h}$.

Flow Cytometry Analysis. Flow cytometry measurements were performed using a BD Accuri C6 device (BD Biosciences). Cells were grown and induced, as described in the whole-cell sensor test section. For each sample, $300 \mu \mathrm{L}$ of cells was taken and then washed with $1 \times$ PBS and diluted as 1 / 100 before the measurement. Forward scatter and side scatter thresholds of 10,000 were used to eliminate any background. 100,000 events were recorded for each measurement, and the resulting data were analyzed with $\mathrm{BD}$ Accuri C6 Plus software (BD Biosciences).

Isolating SARS-CoV-2 RNA from Viral and Patient Samples. Nasopharyngeal swab samples were obtained from the laboratory-confirmed COVID-19 patients and considered as inpatients at Ankara City Hospital, Department of Infectious Diseases and Clinical Microbiology while following proper ethical and clinical regulations. All the samples were collected and stored in viral transfer media following the sample collection guidelines provided by WHO. After heat inactivation at $60{ }^{\circ} \mathrm{C}$ for $90 \mathrm{~min}$, viral RNA isolation is performed using a quick-RNA viral RNA extraction kit (Zymogen) with the standard protocol provided by the supplier. Prior to experiments, all the patient samples and their isolated RNA were tested with the Voliron (Synbiotik LLC) SARS-CoV-2 qRTPCR test kit to identify viral RNA. Viral samples used as a positive control template during the experiments were provided by Prof. Dr. Aykut Özkul, and viral RNA isolated with the same protocol was used for the patient samples.

NASBA Reactions. Overall strategy for the NASBA reactions is adopted from Pardee et al. ${ }^{15}$ As a summary, each 
a)
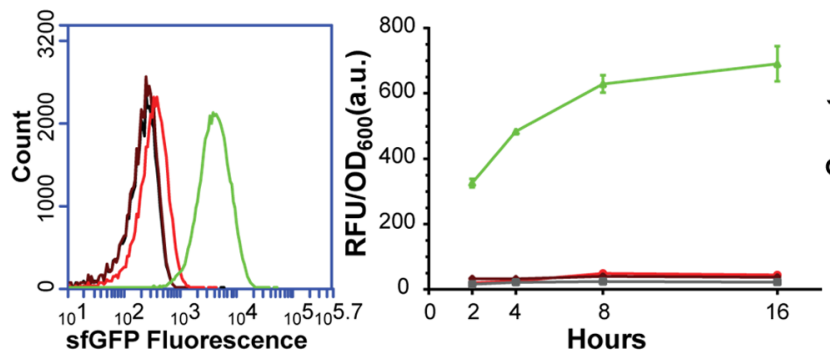

Switch uninduced

- Switch+Trigger uninduced b) S Protein Region Switch, Design 3
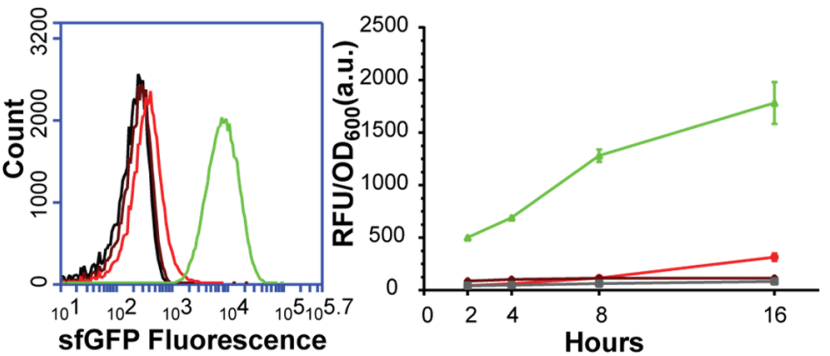

Switch induced

\section{Switch+Trigger induced}

Figure 2. Characterization of best-performing two trigger sequences found in ORF1ab and $S$ protein regions in vivo. For each trigger sequence, four different unique switch sequences were designed and cloned in p15A vector with T7 promoter and sfGFP coding sequence. Trigger sequences were cloned in ColE1 origin vectors with T7 promoter. Both vectors were transformed in E. coli BL21 (DE3) cells and induced with IPTG. Flow cytometry results were taken after $90 \mathrm{~min}$. Meanwhile, cells were monitored for $16 \mathrm{~h}$ via total cell fluorescence measurements using a microplate reader. Cytometer results and total cell fluorescence measurements of designed switch for trigger sequence were found in (a) ORF1ab and (b) $S$ protein regions. For all microplate measurements, the average and standard errors (SE) of three replicates are shown.

reaction constituted of first $1.675 \mu \mathrm{L} 3 \times$ NASBA reaction buffer (Life Sciences, NECB-1-24), $0.8375 \mu \mathrm{L} 6 \times$ nucleotide mix (Life Sciences, NECN-1-24), $0.025 \mu \mathrm{L}$ protector Rnase inhibitor (Roche), and $0.1 \mu \mathrm{L}$ of $12.5 \mu \mathrm{M}$ primer mix, according to the targeted genomic region. After that, $1.125 \mu \mathrm{L}$ of isolated viral RNA was added to the reaction. After incubation at $65{ }^{\circ} \mathrm{C}$ for $2 \mathrm{~min}$ and $41{ }^{\circ} \mathrm{C}$ for $10 \mathrm{~min}, 1.2375 \mu \mathrm{L}$ of NASBA enzyme cocktail (Life Sciences, NEC-1-24) was added to each reaction. Trigger RNA fragments produced by the HiScribe T7 high yield RNA synthesis kit (NEB) and cleaned up with the Monarch RNA cleanup kit (NEB) were used as the positive control during the tests. As the template, T7 controlled trigger plasmids from sensor validation step were used for each toehold switch construct. Same trigger pairs with 750 ng of plasmid DNA were also used for NASBA efficiency tests. Plasmid DNA was used during the tests isolated using the Nucleobond Xtra Midi kit (MN). Note that all the stages of the reaction assembled at $4{ }^{\circ} \mathrm{C}$, and the final NASBA reaction was carried out at $41{ }^{\circ} \mathrm{C}$ for $2 \mathrm{~h}$.

Testing Designs and Samples with Cell-Free Expression Systems. Cell-free testing of designed systems was performed using a commercial cell-free expression kit (NEB, PURExpress) and following the protocol provided by the supplier. For each test sample, isolated switch construct plasmid $(31.5 \mathrm{nM})$ and linear trigger DNA $(0.34 \mathrm{mM})$ were used for testing initial designs. For the measurements with isolated viral RNA, varying amounts of completed NASBA reactions ( 1 or $2 \mu \mathrm{L}$ per reaction) and different concentrations of switch plasmid (500-750 and $1000 \mathrm{ng}$ per reaction) were used as per the previous protocol. Unless otherwise stated, a total volume of $10 \mu \mathrm{L}$ cell-free reactions was used for each test, and the volumes were adjusted with nuclease-free $\mathrm{ddH}_{2} \mathrm{O}$ for changing conditions. Reactions were measured with M5 SpectraMax (Molecular Devices) for 3 or $5 \mathrm{~h}$ at $37{ }^{\circ} \mathrm{C}$ to monitor sfGFP expression by tracking fluorescence emission at $538 \mathrm{~nm}$.

Cross Reactivity Analysis of Best-Performing Switch Sensors. Isolation of viral RNA from the HKU1 viral sample was also carried out with a Zymogen Quick RNA kit. In order to verify isolated HKU1 RNA, a primer set from the literature $^{21}$ was used with SuperScript III Reverse Tran-
scriptase/Taq Enzyme mix and SYBR/ROX reaction mixture using the standard protocol provided by the supplier. Toehold assay for HKU1 cross reactivity was also performed using the SARS-CoV-2 protocol previously mentioned in detail.

Statistical Analyses and Illustrations. Statistical analysis and graphing of data generated in this article were performed with Graphpad Prism 8 software. All the illustrations in this manuscript are created with BioRender.com and Adobe Illustrator by the authors.

Building a Hand-Size Transilluminator. The circuit of the blue light hand illuminator, based on a modified version of Jung et al., ${ }^{22}$ consists of eight $470 \mathrm{~nm}$ light-emitting diodes (LEDs) connected in series to two resistors: one normal and one variable. The normal resistor is used to limit the current that flows into the LEDs, and the variable resistor is used to adjust their brightness. The case of the illuminator was drawn using Solidworks 2019 and then produced with a Makerbot Replicator+ 3D printer using a PLA filament. The case was coated in multiple layers of black spray paint prior to assembly. An orange-transparent cellophane sheet, located in front of sample tubes, is used as the filter. The illuminator powered with three regular $1.5 \mathrm{~V}$ alkaline batteries was used to inspect the result of TXTL reactions.

Data Availability. All data generated and analyzed in this study are available within the article and the Supporting Information is available from the corresponding author upon request.

\section{RESULTS AND DISCUSSION}

We designed synthetic programmable toehold switch sensors to detect SARS-CoV-2-specific genomic regions. Toehold switches are synthetic programmable riboregulators that consist of two main components: a trigger RNA and a switch RNA. The switch RNA structure prevents downstream gene translation through concealment of ribosome-binding site (RBS) and start codon through cis-acting RNA interactions. Following the binding of a trans-acting trigger RNA to the switch, RBS and start codon are relieved, resulting in the activation of downstream gene(s) (Figure 1a). ${ }^{14} \mathrm{LoD}$ of the toehold switch sensor alone might not be clinically sufficient; hence, a primer-based RNA magnification step of the trigger 
a)

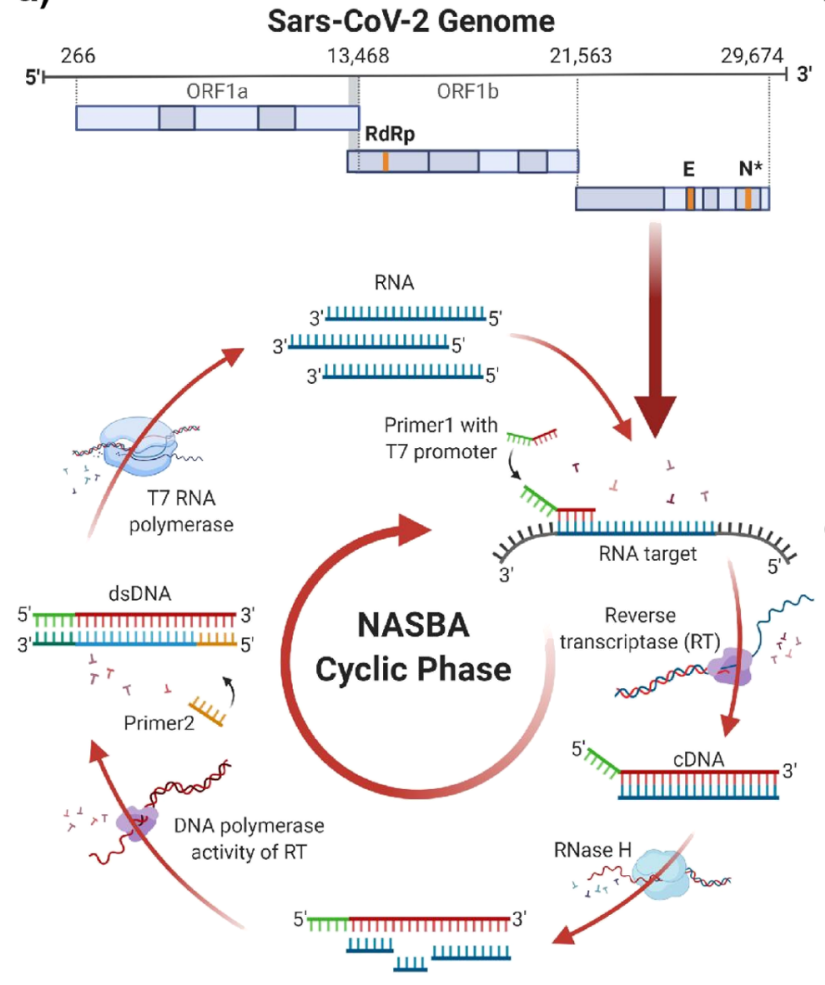

d)

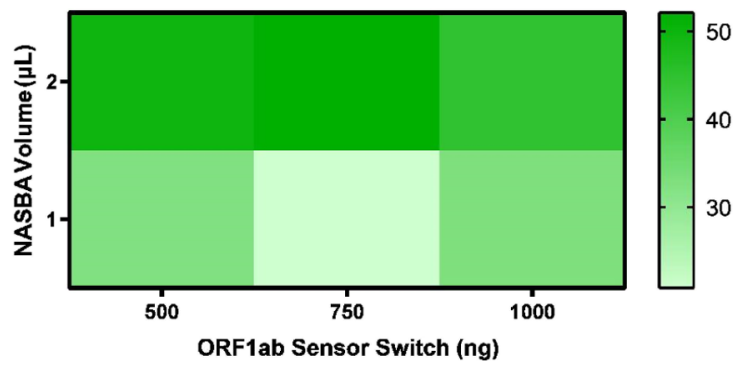

b)
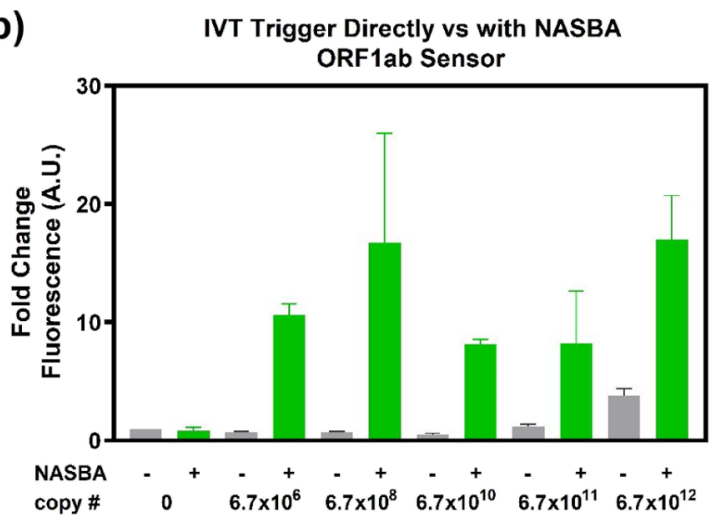

c)

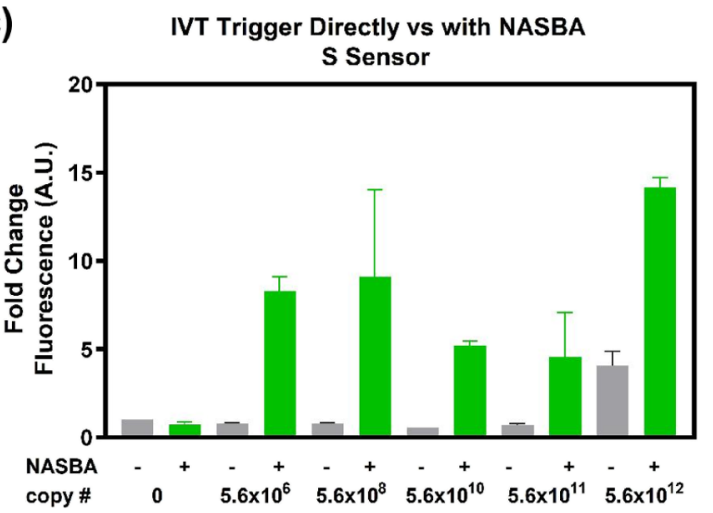

e)

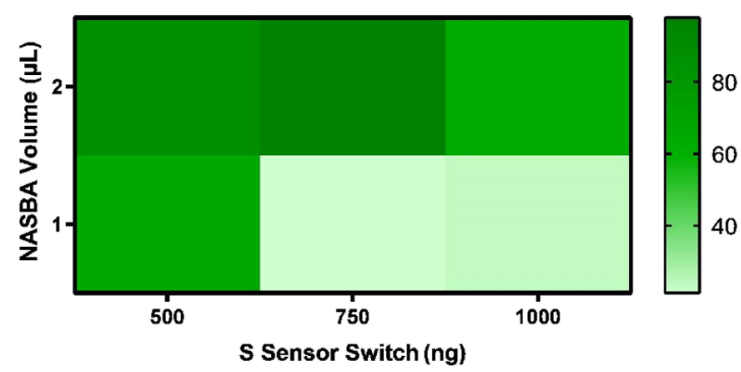

Figure 3. Characterization and optimization of ORFlab trigger/switch and $S$ trigger/switch couples in TXTL reactions. (a) Representation of working principle of the NASBA reaction. In vitro transcribed triggers are either added to TXTL reactions directly or after NASBA reactions, measurements at 60th min are given for (b) ORF1ab and (c) S trigger/switch couple. The average and SE of duplicate experiments are shown. NASBA volume and switch vector amounts are optimized in TXTL reactions. Reactions were monitored for 120 min with 10 min intervals, and endpoint fluorescence measurements of (d) ORF1ab and (e) S trigger/switch couple are given. Representative average and SE of triplicate experiments are shown.

region is a requisite to be included before toehold switch sensor detection. Therefore, to screen amplification compatible candidate trigger regions from the whole SARS-CoV-2 genome (NC_045512.2), NCBI's Primer-BLAST tool was utilized. Free energy of secondary structures of candidate triggers was analyzed with NUPACK. ${ }^{19}$ Candidates that have free energy over $-25.00(\mathrm{kcal} / \mathrm{mol})$ were selected for further analysis. Selected sequences are aligned using BLAST against the genomes of human and close relatives of SARS-CoV-2 to exclusively select SARS-CoV-2-specific triggers. These specific toehold sensors for top five successor triggers are generated using an in silico algorithm, based on multi-tube design of NUPACK $^{23}$ with parameters specified previously. ${ }^{14}$ For each trigger candidate, top-ranked switch designs were checked to prevent in frame stop codons. After all of the described filtering processes, overall, 18 SARS-CoV-2-specific toehold switch sensors were designed with four trigger sequences (Figure $1 \mathrm{~b}$ ).
To test sensors in vivo, fragments for switch and trigger regions were assembled into T7-driven expression p15A and ColE1 origin vectors, respectively. E. coli BL21 (DE3) cells were transformed with both plasmids and induced with IPTG (Figure 1c). Cells were analyzed with a flow cytometer $90 \mathrm{~min}$ after induction (Figures 2 and S1-S4). Additionally, total fluorescence from cells was measured at $2,4,8$, and $16 \mathrm{~h}$ following the IPTG addition to check background signal accumulation (Figures 2 and S1-S4). These in vivo experiments indicated that triggers selected from ORF1ab and $S$ coding regions can be utilized to activate switch-repressed translation with a high signal-to-background ratio (Figure 2a,b).

Based on in vivo assessment of all sensors, the top two bestperforming trigger/switch couples (trigger in ORF1ab region/ design 2 and trigger in $S$ region/design 3) were characterized in the cell-free reaction (Figure S5). In reaction setup, PCR- 
a)
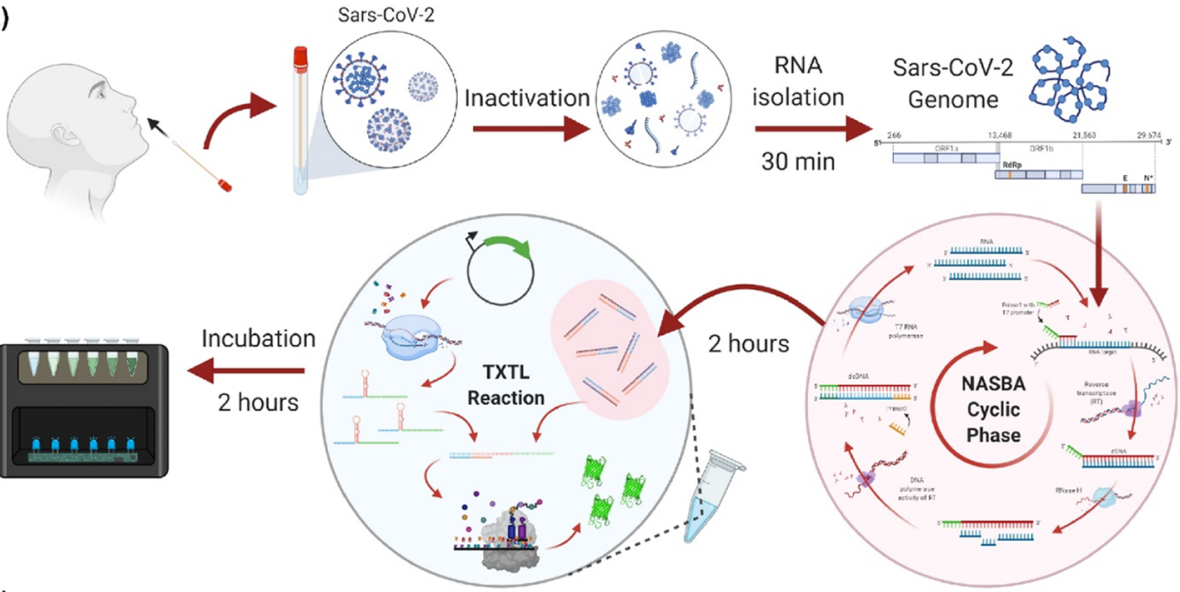

ares En

b)
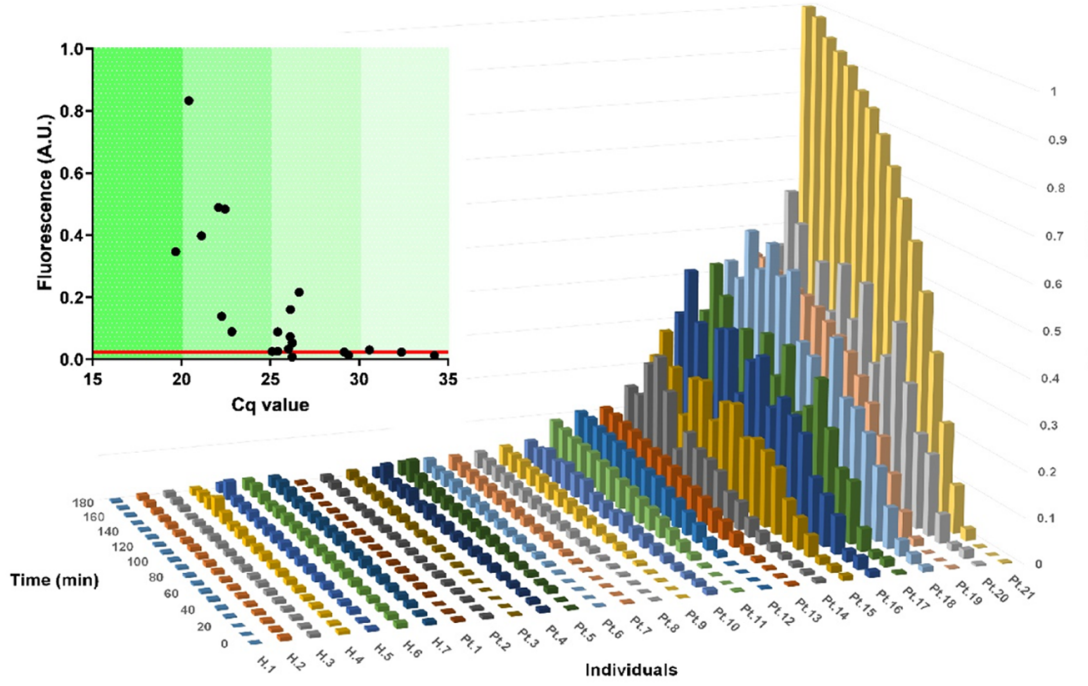

c)

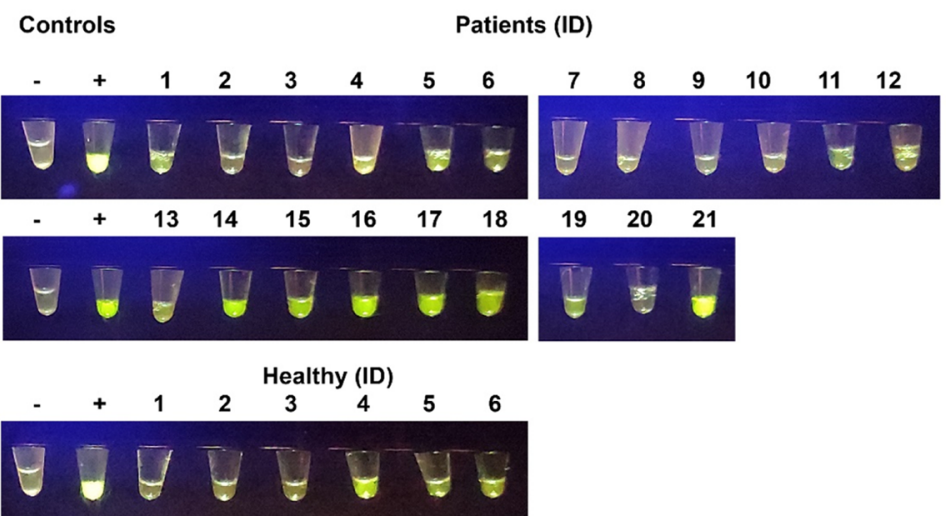

Figure 4. (a) Workflow of the patient sample testing with ORF1ab trigger/switch couple. Viral RNA was isolated from patients' swab samples and incubated in NASBA reactions for $2 \mathrm{~h}$. Once incubation is completed, $2 \mu \mathrm{L}$ of NASBA reaction was added to TXTL cell-free mixture containing $750 \mathrm{ng}$ of switch. Reactions were monitored with the blue light hand illuminator after $120 \mathrm{~min}$ (b) Fluorescence measurements of patient samples with ORFlab trigger/switch couple at different time points, which were monitored for $120 \mathrm{~min}$ with $10 \mathrm{~min}$ intervals on a microplate reader. The abbreviations of $\mathrm{H}$ and $\mathrm{Pt}$ are healthy and patients, respectively. Comparison of patient samples' Cq values and corresponding toehold switch sensor responses of cell-free reactions at 120th min, and the red line shows the sum of average of healthy samples with three times standard deviation. (c) Toehold sensor responses of patient samples are monitored using the in-house built hand illuminator.

amplified triggers with the $\mathrm{T} 7$ promoter region, switch plasmids that bear T7 promoter, corresponding switch sequences and sfGFP coding sequences were used. After the addition of DNA molecules, reactions were transferred into a 384-well plate, and the fluorescence measurement was performed for $6 \mathrm{~h}$ with $10 \mathrm{~min}$ intervals. Both ORF1ab and $\mathrm{S}$ trigger/switch pairs showed similar kinetics in the TXTL reaction in terms of total fluorescence which were saturated after $180 \mathrm{~min}$. Expectedly, no significant increase in the fluorescence signal for "switch alone" circuits was observed. Trigger-activated switch can be distinguished under blue light from mock controls after $40 \mathrm{~min}$ incubation (Figure S5c,d).

Improving SARS-CoV-2 Sensor Sensitivity. The viral loads of clinical specimens were beyond the LoD of toehold 
sensors since the swab samples taken from the upper respiratory tract have been documented to have $6.76 \times 10^{5}$ copies per sample on average. Furthermore, these values can drop to $3.44 \times 10^{5}$ copies per sample if they are taken 5 days after onset of symptoms which lowers the detection rate to $39.93 \%$ with qRT-PCR. ${ }^{24}$ Therefore, incorporation of an isothermal RNA amplification step was necessary to transform our SARS-CoV-2 sensors into a field-ready detection platform. To do so, an isothermal RNA amplification technique, NASBA, was utilized prior to TXTL assay.

To perform NASBA, we have designed primer sets that would specifically amplify and add a T7 promoter to the amplified triggers of ORF1ab and $S$ sensors from the SARSCoV-2 genome (Figure 3a). NASBA reactions were run for 2 $\mathrm{h}$, and $1: 10$ of the completed reactions was added to cell-free reactions. We first demonstrated the value of a prior isothermal amplification step into our detection platform in terms of improving sensitivity. To do so, we compared the overall fluorescent signal levels of TXTL reactions that were ran with the same initial in vitro transcribed trigger RNA concentration; however, in one set same amount of trigger RNA was subjected to NASBA amplification before adding it to the TXTL reaction (Figure 3b,c).

After that, to optimize the signal levels of sensors when tested with clinical specimens, the patient samples that have been all tested positive previously but had varying $\mathrm{Cq}$ values have been pooled. The NASBA reactions were performed with the isolated RNA obtained from the pooled patient samples. To determine the best-performing trigger to switch the ratio in TXTL reactions, a combinatorial experiment was set up. Here, addition of $1: 10$ and 1:5 of NASBA reactions was tested against 500, 750, and $1000 \mathrm{ng}$ of switch plasmids in cell-free reactions for both ORF1ab and S sensors, respectively (Figures 3d,e and S6). An increase in the signal levels was observed for all cases where the NASBA volume was increased in the cellfree reactions. Since there was not a linear response to increasing switch concentrations, it was thought that the concentrations of triggers and switches in the TXTL reactions may not be the only contributing factor of the reaction efficiency. Transcription efficiency in the cell-free reactions may have also been affected by the availability of free T7 RNAP. Therefore, the best-performing switch to the NASBA ratio was determined to be moderate levels of switch $(750 \mathrm{ng})$ to a high volume of NASBA reactions $(2 \mu \mathrm{L})$ for further experiments.

Monitoring Human Samples with Portable, Low-Cost PoC Device. Collected clinical swab samples were stored in viral lysis and transfer media and then inactivated. Afterward, viral RNA was isolated to be added to the NASBA reaction to amplify trigger regions specific to the SARS-CoV-2 genome. Amplified trigger regions are added to TXTL reactions containing switch plasmids with the optimum ratios, as determined previously (750 ng switch with $2 \mu \mathrm{L}$ of NASBA in $10 \mu \mathrm{L}$ reactions). TXTL reactions are incubated for $2 \mathrm{~h}$ at $37^{\circ} \mathrm{C}$, and reactions are then visualized with the in-house built blue-light illuminator (Figure 4a).

Pooled patient RNA samples used for NASBA volume to switch amount optimization have demonstrated that the system is applicable to clinical samples. Moreover, both $S$ and ORF1ab switch sensors are shown not to give significant results to HKU viral RNA under the same experimental conditions (Figure S7). First, to minimize the duration of reaction time to get a detectable signal change, RNA samples isolated from patients were tested with NASBA-TXTL assay in kinetic time measurement on a microplate reader (Figure $4 \mathrm{~b}$ ). With an instrument as sensitive as a microplate reader, the signal change is detectable at 60th min but with naked eye through the in-house built illuminator at 90th to 120th min. Second, to detect LoD minima, toehold sensor responses of cell-free reactions at 120 th $\mathrm{min}$ are compared with quantification cycle $(\mathrm{Cq})$ values obtained from qRT-PCR tests (Table S2). Toehold switch sensor response is inversely correlated with $\mathrm{Cq}$ values, parallel to our expectations (Figure 4b). Then, patient samples are monitored using the hand illuminator for the visibility of their toehold sensor responses, as seen in Figure 4c. Consequently, COVID-19 positive individuals' samples can be visualized with our proposed detection platform. Altogether, these findings suggest that the applicability of our designed diagnostic platform can be used as a quick responsive and portable tool as it does not require expensive instruments, special laboratory environments, or experts.

\section{CONCLUSIONS}

We have successfully developed a de novo-designed riboregulator system to detect the presence of the SARS-CoV-2specific genomic regions. To develop the diagnostic system, an in vitro synthetic biology tool has been employed. More than 400 riboregulator designs have been screened in silico in terms of their stability, energy, applicability to NASBA reactions, and specificity to SARS-CoV-2. After selecting the top five promising candidates, trigger/switch couples are tested in vivo and then candidates with low background and high fold changes are analyzed using in vitro TXTL assay. As a result, the most promising candidates, a region from the spike protein $(S)$ and ORFlab region of the SARS-CoV-2 viral genome, have been selected for further optimization to be used in the diagnostic device. First, $S$ and ORF1ab sensors' response to different copy numbers with and without amplification steps prior to TXTL assay was investigated. The sensor targeting the ORFlab region showed higher fold change at low copy numbers compared to the $S$ sensor with prior NASBA reaction. However, the $S$ sensor's response was higher when TXTL reaction components (NASBA volume and switch plasmid amount) are optimized. Considering that the detection of low copy numbers is pivotal in the diagnosis of infected individuals, the studies were continued with the ORFlab sensor. Healthy and patient samples (qRT-PCR negative and positive, respectively) obtained from Ankara City Hospital have been tested with our proposed diagnostic platform. The kinetic measurement of the cell-free systems showed that this system can detect the viral genome parts in 60 min via highly sensitive detectors of a microplate reader, though eye visibility with blue light illumination with a transparent orange filter is achieved in $2 \mathrm{~h}$. Even though the sensors have been shown to be sensitive to low copy numbers using IVT assay, the results are not reflected in human samples. The minimal copy number distinguished with our proposed system is determined to be as low as 1800 copies. Changing the primer pairs to shift the trigger region while covering the recognition site on its corresponding switch is an alternative. ${ }^{17}$ These findings suggest that further optimization of the NASBA primers would increase the sensitivity when applied to human samples.

Considering the requirements of the steps in the diagnostic system, it is a promising platform due to its easy application without the need for a laboratory environment, an expert in the 
field, or special and highly expensive equipment. Duration of an RNA isolation step can be decreased to several minutes with the use of functionalized magnetic beads. The NASBA reaction can be reduced to 90 to $60 \mathrm{~min}$, with the possibility of lower signal levels with shortened response time. Similarly, the incubation time of TXTL reaction can be shortened, whereas the eye visibility of the results might not be applicable, and the integration of highly sensitive sensors into the PoC device will increase the cost to a great extent.

Another crucial aspect when developing a PoC device is the cost of the end product. As in our calculations, isothermal nucleic acid amplification and cell-free reaction used in the test cost 0.89 cent per sample based on the current price on the NEB website, yet it can be further decreased with mass production. In addition, the monitoring device can be manufactured for as low as $30 \$$, according to prototyping companies that we contacted in Turkey.

To sum up, although vaccination has been started for many countries, achieving herd immunity requires a substantial time based on the vaccination rate. Moreover, many underdeveloped countries will not have access to vaccines in the near future, making mass testing the only option to suppress the spread. Compared with other commercially available tests such as qRT-PCR or serological assays, the de novo-designed riboregulator-based sensing platforms have more advantages for detection of the SARS-CoV-2 genome. First, considering the ease of engineering, our proposed system can be redesigned/engineered to sense new strains of SARS-CoV-2 in the midst of continuing pandemic and other pathogens in the future. Second, designing and testing of sensor candidates can be completed within a week. Third, the steps in the diagnostic system are isothermal; hence, there are no needs for specific equipment, laboratory environments, or experts, so the system can be used as a portable diagnostic tool. Fourth, the cost of each test is lower than a dollar, and also the hand illuminator device can be used multiple times. Taken together, albeit the LoD of our proposed test is not as high as that of qRT-PCR, the implementation into a quick-responsive, portable, and low-cost PoC device is promising.

\section{ASSOCIATED CONTENT}

\section{SI Supporting Information}

The Supporting Information is available free of charge at https://pubs.acs.org/doi/10.1021/acs.analchem.1c00886.

Performance of the designed sensing switches to the trigger sequence found in ORF1ab, S protein, ORF678, and $\mathrm{M}$ protein region in E. coli BL21 (DE3); characterization of $S$ trigger/switch and ORF1ab trigger/switch couples in the TXTL reaction; characterization of NASBA volume and switch plasmid amount in TXTL reactions; cross reactivity analysis of ORF1ab trigger/switch and $S$ trigger/switch couples with HKU1 viral RNA; target domains and the lengths of de novodesigned riboregulators specific to SARS-CoV-2 used in this study; and qRT-PCR measurements of patient samples with a Voliron qPCR kit (PDF)

\section{AUTHOR INFORMATION}

\section{Corresponding Author}

Urartu Özgür Şafak Şeker - UNAM-National

Nanotechnology Research Center, Bilkent University, 06800

Ankara, Turkey; Institute of Materials Science and
Nanotechnology, Bilkent University, 06800 Ankara, Turkey; ○ orcid.org/0000-0002-5272-1876; Email: urartu@ bilkent.edu.tr

\section{Authors}

İlkay Çisil Köksaldı - UNAM-National Nanotechnology Research Center, Bilkent University, 06800 Ankara, Turkey; Institute of Materials Science and Nanotechnology, Bilkent University, 06800 Ankara, Turkey

Sıla Köse - UNAM-National Nanotechnology Research Center, Bilkent University, 06800 Ankara, Turkey; Institute of Materials Science and Nanotechnology, Bilkent University, 06800 Ankara, Turkey

Recep Erdem Ahan - UNAM-National Nanotechnology Research Center, Bilkent University, 06800 Ankara, Turkey; Institute of Materials Science and Nanotechnology, Bilkent University, 06800 Ankara, Turkey

Nedim Haclosmanoğlu - UNAM-National Nanotechnology Research Center, Bilkent University, 06800 Ankara, Turkey; Institute of Materials Science and Nanotechnology, Bilkent University, 06800 Ankara, Turkey

Ebru Şahin Kehribar - UNAM-National Nanotechnology Research Center, Bilkent University, 06800 Ankara, Turkey; Institute of Materials Science and Nanotechnology, Bilkent University, 06800 Ankara, Turkey

Murat Alp Güngen - UNAM-National Nanotechnology Research Center, Bilkent University, 06800 Ankara, Turkey; Institute of Materials Science and Nanotechnology, Bilkent University, 06800 Ankara, Turkey

Aliye Baştug - Department of Infectious Diseases and Clinical Microbiology, Health Science University Turkey, Ankara City Hospital, 06800 Ankara, Turkey

Bedia Dinç - Department of Infectious Diseases and Clinical Microbiology, Health Science University Turkey, Ankara City Hospital, 06800 Ankara, Turkey

Hürrem Bodur - Department of Infectious Diseases and Clinical Microbiology, Health Science University Turkey, Ankara City Hospital, 06800 Ankara, Turkey

Aykut Özkul - Faculty of Veterinary Medicine, Department of Virology, Ankara University, 06110 Ankara, Turkey; Biotechnology Institute, Ankara University, 06135 Ankara, Turkey

Complete contact information is available at:

https://pubs.acs.org/10.1021/acs.analchem.1c00886

\section{Author Contributions}

\#i.Ç.K., S.K., R.E.A., N.H., and E.Ş.K. contributed equally. Notes

The authors declare the following competing financial interest(s): U.O.S.S., I.C.K., S.K., R.E.A., N.H., and E.S.K. filed a patent application.

\section{ACKNOWLEDGMENTS}

We thank TÜBİTAK COVID-19 Platform and Bilkent University UNAM for partial financial support. We also thank Can Güven from Bilkent University UNAM for his help with 3D printing of the PoC device. U.Ö.Ş.Ş. also thanks Ceyda, Ruzgar, and Deniz for their invaluable support and understanding during the COVID-19 pandemic lockdown.

\section{REFERENCES}

(1) Li, S.-r.; Tang, Z.-j.; Li, Z.-h.; Liu, X. Eur. J. Clin. Microbiol. Infect. Dis. 2020, 39, 1021. 
(2) Hoffmann, M.; Kleine-Weber, H.; Schroeder, S.; Krüger, N.; Herrler, T.; Erichsen, S.; Schiergens, T. S.; Herrler, G.; Wu, N.-H.; Nitsche, A. Cell 2020, 181, 271.

(3) Baj, J.; Karakuła-Juchnowicz, H.; Teresiński, G.; Buszewicz, G.; Ciesielka, M.; Sitarz, E.; Forma, A.; Karakuła, K.; Flieger, W.; Portincasa, P. J. Clin. Med. 2020, 9, 1753.

(4) Cheng, M.-Y.; Hsih, W.-H.; Ho, M.-W.; Chou, C.-H.; Lin, P.-C.; Chi, C.-Y.; Liao, W.-C.; Chen, C.-Y.; Leong, L.-Y.; Tien, N.; et al. J. Microbiol., Immunol. Infect. 2020, 53 (3), 459-466.

(5) Meyerowitz, E. A.; Richterman, A.; Bogoch, I. I.; Low, N.; Cevik,

M. Lancet Infect. Dis. 2021, 21, No. e163.

(6) Morales-Narváez, E.; Dincer, C. Biosens. Bioelectron. 2020, 163, 112274.

(7) Loeffelholz, M. J.; Tang, Y.-W. Emerging Microbes Infect. 2020, 9, 747-756.

(8) Esbin, M. N.; Whitney, O. N.; Chong, S.; Maurer, A.; Darzacq, X.; Tjian, R. RNA 2020, 26, 771.

(9) FDA. https://www.fda.gov/medical-devices/coronavirusdisease-2019-covid-19-emergency-use-authorizations-medicaldevices/in-vitro-diagnostics-euas-molecular-diagnostic-tests-sars-cov2, 2020.

(10) Broughton, J. P.; Deng, X.; Yu, G.; Fasching, C. L.; Servellita, V.; Singh, J.; Miao, X.; Streithorst, J. A.; Granados, A.; SotomayorGonzalez, A. Nat. Biotechnol. 2020, 38, 870.

(11) Park, G.-S.; Ku, K.; Baek, S.-H.; Kim, S.-J.; Kim, S. I.; Kim, B.T.; Maeng, J.-S. J. Mol. Diagn. 2020, 22, 729.

(12) Lu, R.; Wu, X.; Wan, Z.; Li, Y.; Jin, X.; Zhang, C. Int. J. Mol. Sci. 2020, 21, 2826.

(13) Fozouni, P.; Son, S.; Díaz de León Derby, M.; Knott, G. J.; Gray, C. N.; D’Ambrosio, M. V.; Zhao, C.; Switz, N. A.; Kumar, G. R.; Stephens, S. I.; Boehm, D.; Tsou, C.-L.; Shu, J.; Bhuiya, A.; Armstrong, M.; Harris, A. R.; Chen, P.-Y.; Osterloh, J. M.; MeyerFranke, A.; Joehnk, B.; et al. Cell 2021, 184, 323-333.

(14) Green, A. A.; Silver, P. A.; Collins, J. J.; Yin, P. Cell 2014, 159, 925-939.

(15) Pardee, K.; Green, A. A.; Takahashi, M. K.; Braff, D.; Lambert, G.; Lee, J. W.; Ferrante, T.; Ma, D.; Donghia, N.; Fan, M. Cell 2016, 165, 1255-1266.

(16) Pardee, K.; Green, A. A.; Ferrante, T.; Cameron, D. E.; DaleyKeyser, A.; Yin, P.; Collins, J. Cell 2014, 159, 940-954.

(17) Takahashi, M. K.; Tan, X.; Dy, A. J.; Braff, D.; Akana, R. T.; Furuta, Y.; Donghia, N.; Ananthakrishnan, A.; Collins, J. Nat. Commun. 2018, 9, No. 3347.

(18) de Puig, H.; Bosch, I.; Collins, J. J.; Gehrke, L. Annu. Rev. Biomed. Eng. 2020, 22, 371-386.

(19) Zadeh, J. N.; Steenberg, C. D.; Bois, J. S.; Wolfe, B. R.; Pierce, M. B.; Khan, A. R.; Dirks, R. M.; Pierce, N. A. J. Comput. Chem. 2011, 32, 170-173.

(20) Olson, E. J.; Hartsough, L. A.; Landry, B. P.; Shroff, R.; Tabor, J. Nat. Methods 2014, 11, 449-455.

(21) Cordes, A. K.; Rehrauer, W. M.; Accola, M. A.; Wölk, B.; Hilfrich, B.; Heim, A. J. Med. Virol. 2020, 93, 4438.

(22) Jung, J. K.; Alam, K. K.; Verosloff, M. S.; Capdevila, D. A.; Desmau, M.; Clauer, P. R.; Lee, J. W.; Nguyen, P. Q.; Pastén, P. A.; Matiasek, S. J. Nat. Biotechnol. 2020, 38, 1451.

(23) Wolfe, B. R.; Porubsky, N. J.; Zadeh, J. N.; Dirks, R. M.; Pierce, N. A. J. Am. Chem. Soc. 2017, 139, 3134-3144.

(24) Wölfel, R.; Corman, V. M.; Guggemos, W.; Seilmaier, M.; Zange, S.; Müller, M. A.; Niemeyer, D.; Jones, T. C.; Vollmar, P.; Rothe, C.; Hoelscher, M.; Bleicker, T.; Brünink, S.; Schneider, J.; Ehmann, R.; Zwirglmaier, K.; Drosten, C.; Wendtner, C. Nature 2020, 581, 465-469. 